\title{
Cooper channel and the singularities in the thermodynamics of a Fermi liquid
}

\author{
Andrey V. Chubukov ${ }^{1}$ and Dmitrii L. Maslov ${ }^{2}$ \\ ${ }^{1}$ Department of Physics, University of Wisconsin-Madison, \\ 1150 Univ. Ave., Madison, WI 53706-1390 and \\ ${ }^{2}$ Department of Physics, University of Florida, P. O. Box 118440, Gainesville, FL 32611-8440
}

\begin{abstract}
We analyze how the logarithmic renormalizations in the Cooper channel affect the non-analytic temperature dependence of the specific heat coefficient $\gamma(T)-\gamma(0)=A(T) T$ in a $2 \mathrm{D}$ Fermi liquid. We show that $A(T)$ is expressed exactly in terms of the fully renormalized backscattering amplitude which includes the renormalization in the Cooper channel. In contrast to the $1 \mathrm{D}$ case, both charge and spin components of the backscattering amplitudes are subject to this renormalization. We show that the logarithmic renormalization of the charge amplitude vanishes for a flat Fermi surface, when the system becomes effectively one-dimensional.
\end{abstract}

PACS numbers:

Non-analytic behavior of the thermodynamic parameters of a Fermi liquid has a been of interest since the early days of Fermi-liquid theory, when it was found that for $D=3$ the specific heat coefficient $\gamma(T)=C(T) / T$ has a non-analytic temperature dependence $\delta \gamma(T)=$ $\gamma(T)-\gamma(0) \propto T^{2} \ln T$ [1]. The issue of non-analyticities has been revived in recent years, following the work by Belitz, Kirkpatrick, and Vojta 2], who found that the same type of logarithmic behavior holds for the spin susceptibility at a finite momentum $q$ in three dimensions (3D) $\delta \chi_{s}(q) \propto q^{2} \ln q$.

Non-analyticities are stronger in two than in three dimensions: $\delta \gamma(T, H)$ and $\delta \chi_{s}(T, q, H)$ are linear functions of their respective variables 3, 4, 5, 6, 7, 8, 9, 10, 11, 12, 13, 14, 15]. In Refs. [11, 12], it was argued that the non-analytic part $\delta \gamma(T)$ comes from one-dimensional scattering processes embedded in a $2 \mathrm{D}$ phase space [14] and that $\delta \gamma(T)$ is expressed via the charge and spin components of the exact scattering amplitude with zero total momentum $f(\theta=\pi)$ ("backscattering amplitude")

$$
\delta \gamma(T)=-\frac{3 \zeta(3)}{2 \pi\left(v_{F}^{*}\right)^{2}}\left[f_{c}^{2}(\pi)+3 f_{s}^{2}(\pi)\right] T .
$$

Validity of Eq.(1) was verified perturbatively in Ref. [11] by calculating $\delta \gamma(T)$ and $f_{c, s}(\pi)$ independently to third and second order in the interaction, respectively, and checking that Eq. (11) holds. That analysis, however, was incomplete - it included the renormalizations in the particle-hole channel but neglected the renormalizations in the Cooper channel. Refs. [11, 12] conjecturedwithout proof- that Eq.(11) still holds if the Cooper renormalizations are included into $f_{c, s}(\pi)$.

The Cooper renormalization of the backscattering amplitudes is an essential ingredient of the theory, particularly in the limit $T \rightarrow 0$. The argument is that the backscattering amplitude

$$
f_{\alpha \beta ; \gamma \delta}(\pi)=f_{c}(\pi) \delta_{\alpha \beta} \delta_{\gamma \delta}+f_{s}(\pi) \tilde{\sigma}_{\alpha \beta} \cdot \tilde{\sigma}_{\gamma \delta}
$$

is expressed in terms of two full vertices with zero total momentum and either zero or $2 k_{F}$ momentum trans- fer $\Gamma(\mathbf{k},-\mathbf{k} ; \mathbf{k},-\mathbf{k}) \equiv \Gamma(\mathbf{k}, \mathbf{k})$ and $\Gamma(\mathbf{k},-\mathbf{k} ;-\mathbf{k}, \mathbf{k}) \equiv$ $\Gamma(\mathbf{k},-\mathbf{k})$ :

$f_{c}(\pi)=\frac{m}{\pi}\left[\Gamma(\mathbf{k}, \mathbf{k})-\frac{1}{2} \Gamma(\mathbf{k},-\mathbf{k})\right], f_{s}(\pi)=-\frac{m}{2 \pi} \Gamma(\mathbf{k},-\mathbf{k})$

Equivalently, each of these two vertices can be expressed via the fully renormalized Cooper vertex with either zero or $2 k_{F}$ momentum transfer: $\Gamma(\mathbf{k}, \mathbf{k})=$ $\Gamma^{C}(0), \Gamma(\mathbf{k},-\mathbf{k})=\Gamma^{C}\left(2 k_{F}\right)$. Therefore, these vertices can be expressed via the partial components $J_{n}^{C}$ of the irreducible interaction in the Cooper channel as [20]

$$
\Gamma^{C}(0), \Gamma^{C}\left(2 k_{F}\right)=\sum_{n}( \pm 1)^{n} \frac{J_{n}^{C}}{1+\frac{m}{2 \pi} J_{n}^{C} \ln \frac{E_{F}}{T}} .
$$

At low temperatures, all terms in the sum scale as $\left(\ln E_{F} / T\right)^{-1}$, so that $\Gamma(k, k)$ and $\Gamma(k,-k)$ should, in general, be reduced by the same logarithmic factor. Whether this logarithmic renormalization affects the backscattering amplitudes and $\delta \gamma(T)$ is a more subtle issue which is to be addressed by a direct calculation.

The 1D case serves as a good example here. Both $\Gamma(k, k)$ and $\Gamma(k,-k)$ are logarithmically renormalized in $1 \mathrm{D}$, yet, these renormalizations only affect the spin channel, but cancel out in the charge channel [16, 17, 18, 19]. As a result, the specific heat remains linear in $T$ at the lowest $T$. This agrees with $1 \mathrm{D}$ bosonization according to which charge excitations are described by a free Gaussian theory, whereas the spin channel for the case a repulsive interaction contains a marginally irrelevant perturbation that causes a logarithmic flow of the spin amplitude. The issue that we address in this paper is whether this situation occurs only in 1D or in higher dimensions as well.

The interplay between the logarithmic renormalization of the interaction and the behavior of specific heat coefficient in $2 \mathrm{D}$ has recently been considered by Aleiner and Efetov (AE) 15] (for a subsequent analysis see [8, 9]). AE invented an elegant supersymmetric method to treat the problem in arbitrary D by integrating out fermions and expressing the low-energy ac- 
tion solely in terms of the low-energy collective bosonic modes. They found that the spin contribution to $\delta \gamma(T)$ is affected by the Cooper renormalization and behaves as $T(\ln |\ln T| / \ln T)^{2}$ in the limit of $T \rightarrow 0$. They treated the charge component in the eikonal approximation which neglects the curvature of the Fermi surface, and found that, in this approximation, the charge component remains unrenormalized.

In this communication, we re-consider this issue. We evaluate $\delta \gamma(T)$ explicitly to third order in the interaction $U(q)$, including Cooper renormalizations, and also evaluate the spin and charge components of the backscattering amplitude to second order in $U(q)$. We find that, in contrast to the $1 \mathrm{D}$ case, both $f_{s}(\pi)$ and $f_{c}(\pi)$ undergo logarithmic renormalization in 2D. We also find that Eq. (11) still holds when $U(q)$ is re-expressed in terms of $f_{s}(\pi)$ and $f_{c}(\pi)$. This result agrees with the conjecture made in Refs. [11, 12]. For a short-range interaction, we find that the spin contribution to $\delta \gamma(T)$ scales as $(1 / \ln T)^{2}$, while the charge contribution scales as $(\ln |\ln T| / \ln T)^{2}$. As a result, if the system remains in the normal state down to very low $T, \delta \gamma(T)$ would be dominated by charge fluctuations and behaved as $\delta \gamma(T) \propto T(\ln |\ln T| / \ln T)^{2}$. The normal state behavior in $2 \mathrm{D}$, however, exists only down to the temperature $T_{p}$ of the Kohn-Luttinger instability towards $p$-wave pairing [22, 23]. We analyzed $\delta \gamma(T)=A(T) T$ near $T_{p}$ and found that $A(T)$ diverges as $\left(T-T_{p}\right)^{-2}$.

As a simple check, we verified that the same procedure that we used for calculating the scattering amplitudes in $2 \mathrm{D}$ reproduces the known result in $1 \mathrm{D}$, namely, the cancellation of the logarithmic renormalizations in the charge channel [16, 17, 18, 19]. This comparison helps to see where exactly the cases of $D>1$ and $D=1$ differ: for $D>1$, only the Cooper channel is logarithmic; hence there is no cancellation between the Cooper and particle-hole channels, and $f_{c}(\pi)$ is renormalized along with $f_{s}(\pi)$. For $D=1$, both particle-hole and particleparticle renormalizations are logarithmic, and the two cancel each other in the charge channel. To further emphasize this point, we also considered the 2D case with a non-circular Fermi surface and demonstrated that when the Fermi surface becomes flat, the particle-hole renormalization becomes logarithmic and the Cooper and particle-hole renormalizations again cancel each other in the charge channel.

We believe that it is the effect of the curvature that is responsible for the difference between our result and that of AE.

We begin with the 2D case and a circular Fermi surface. To second order in $U(q)$, the diagrams for the thermodynamic potential $\Xi$ contain two fermionic bubbles $\Pi(q, \Omega)$. To this order, the non-analyticity comes from the square of the dynamic part of $\Pi(q, \Omega)$ which contains the term $\Omega^{2} / q^{2}$ at $v_{F} q \gg|\Omega|[9,11,12]$. Because the momentum integral is logarithmic in 2D, the second-order thermo-
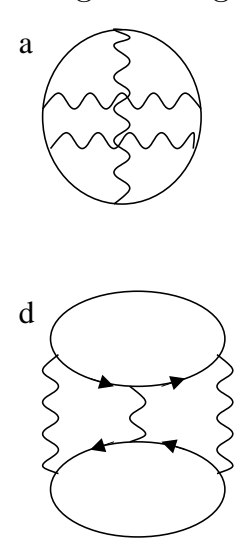

b

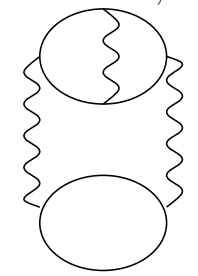

e

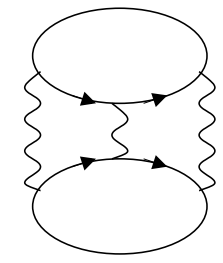

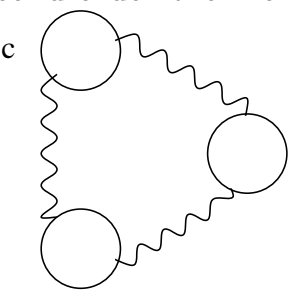

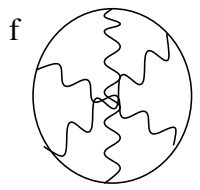

FIG. 1: Diagrams for the thermodynamic potential to third order in the interaction.

dynamic potential

$$
\Xi \sim U^{2} T \sum_{\Omega} \int d^{2} q \Pi^{2} \sim U^{2} T \sum_{\Omega} \Omega^{2} \ln |\Omega| \sim U^{2} T^{3}
$$

contains a universal $T^{3}$ term, which gives rise to a $O(T)$ term in $\delta \gamma$. It was shown in Refs. [9, 11, 12] that the momenta carried by fermions in the two bubbles, $\mathbf{k}_{1}, \mathbf{k}_{2}, \mathbf{k}_{3}$, and $\mathbf{k}_{4}$, are correlated in such a way that $\mathbf{k}_{1} \approx \mathbf{k}_{2} \approx-\mathbf{k}_{3} \approx-\mathbf{k}_{4}$. These four fermions can then be re-arranged either into a convolution of two particle-hole bubbles $\Pi_{p h}(\Omega, q)$ or two particle-particle bubbles [6, 9] $\Pi_{p p}(\Omega, q)$; the term $\Omega^{2} / q^{2}$ is produced regardless of the choice.

Relevant third-order diagrams for $\Xi$ are shown in Fig 1 . They contain either three particle-hole or three particleparticle bubbles. It can be shown that the non-analytic terms in $\delta \gamma(T)$ come from the terms containing the products of two dynamic and one static parts of these bubbles. Two dynamic parts of the bubbles produce $\Omega^{2} / q^{2}$ term, which is the source of non-analyticity, whereas the third bubble renormalizes static backscattering vertex. If the third bubble is a particle-hole one, the renormalized vertex is a constant. The momentum integration then yields $\Omega^{2} \ln |\Omega|$, and subsequent frequency summation gives rise to the $T^{3}$-term in $\Xi$. In the third bubble is a particle-particle one, the renormalized vertex contains an additional factor of $\ln q$. This changes the result of the momentum integration to $\Omega^{2} \ln ^{2}|\Omega|$ [note an extra $\ln |\Omega|$ as compared to Eq.(44)] and gives rise to a $T \ln T$-term in $\delta \gamma(T)$.

The contributions from diagrams (a-d) have already been presented in [11], diagrams (e) and (f) were not considered there. For completeness, we present the results for all third-order diagrams. We have 21] 


$$
\begin{aligned}
& \Xi_{3 a}=-\left(u_{0}\left\langle u_{\theta} u_{\pi-\theta}\right\rangle+2 u_{0} u_{\pi}\left\langle u_{\theta}\right\rangle+u_{\pi}\left\langle\left\langle u_{\theta}^{2}\right\rangle\right\rangle+2 u_{0} u_{\pi}\left\langle\left\langle u_{\theta}\right\rangle\right\rangle\right) K, \quad \Xi_{3 b}=\left(4 u_{0}^{2}\left\langle u_{\theta}\right\rangle+2 u_{0}^{2} u_{\pi}\right) K \\
& +\left[4 u_{\pi}^{2}\left\langle\left\langle u_{\theta}\right\rangle\right\rangle+2 u_{\pi}^{2} u_{0}\langle\langle 1\rangle\rangle\right] K, \quad \Xi_{3 c}=-4\left[u_{0}^{3}+u_{\pi}^{3}\langle\langle 1\rangle\rangle\right] K, \quad \Xi_{3 d}=2 u_{\pi}\left\langle u_{\theta} u_{\pi-\theta}\right\rangle K+2 u_{0}\left\langle\left\langle u_{\theta}^{2}\right\rangle\right\rangle K, \\
& \Xi_{3 e}=-\left(2 u_{0}\left\langle u_{\theta}^{2}\right\rangle+2 u_{\pi}\left\langle u_{\theta} u_{\pi-\theta}\right\rangle\right) K \ln \frac{E_{F}}{T}, \Xi_{3 f}=\left(u_{0}\left\langle u_{\theta} u_{\pi-\theta}\right\rangle+u_{\pi}\left\langle u_{\theta}^{2}\right\rangle\right) K \ln \frac{E_{F}}{T}
\end{aligned}
$$

where $K \equiv \zeta(3) T^{3} / \pi v_{F}^{2}$ and $u_{\theta}=(m / 2 \pi) U\left(2 k_{F} \sin \theta / 2\right)$, such that $u_{0}=(m / 2 \pi) U(0)$ and $u_{\pi}=(m / 2 \pi) U\left(2 k_{F}\right)$.
Quantities denoted as $\left\langle g_{\theta}\right\rangle$ and $\left\langle\left\langle g_{\theta}\right\rangle\right\rangle$ are $\left(g_{\theta}=u_{\theta}, u_{\theta}^{2}\right.$, and 1):

$\left\langle g_{\theta}\right\rangle=-\frac{2 \pi}{m} \int \frac{d^{2} l d \omega}{(2 \pi)^{3}} g(|\mathbf{1}|) G_{\mathbf{1}+\mathbf{k}_{F}}^{2}=\int_{0}^{\pi} \frac{d \theta}{\pi} g_{\theta} ;\left\langle\left\langle g_{\theta}\right\rangle\right\rangle=-\frac{2 \pi}{m} \int \frac{d^{2} l d \omega}{(2 \pi)^{3}} g(|\mathbf{1}|) G_{\mathbf{1}+\mathbf{k}_{F}} G_{\mathbf{l}-\mathbf{k}_{F}}=\int_{0}^{\pi} \frac{d \theta}{\pi} g_{\theta} \cos \frac{\theta}{2} \ln \left(\cot \frac{\theta}{4}\right)$.

For a circular Fermi surface, $\langle\langle 1\rangle\rangle=1$.

Combining $\Xi_{3}$ with the second-order result $\Xi_{2}=\left(u_{0}^{2}+\right.$ $\left.u_{\pi}^{2}-u_{0} u_{\pi}\right) K$ [1] we obtain a complete result for $\delta \gamma(T)$ to third order in the interaction

$$
\begin{aligned}
& \delta \gamma(T)=-\left[u_{0}^{2}+u_{\pi}^{2}-u_{0} u_{\pi}-4 u_{0}^{3}+2 u_{0}^{2} u_{\pi}+\left(4 u_{0}^{2}-2 u_{0} u_{\pi}\right)\left\langle u_{\theta}\right\rangle-\left(u_{0}-2 u_{\pi}\right)\left\langle u_{\theta} u_{\pi-\theta}\right\rangle+\left(-4 u_{\pi}^{3}+2 u_{0} u_{\pi}^{2}\right)\langle\langle 1\rangle\rangle\right. \\
& \left.+\left(4 u_{\pi}^{2}-2 u_{0} u_{\pi}\right)\left\langle\left\langle u_{\theta}\right\rangle\right\rangle-\left(u_{\pi}-2 u_{0}\right)\left\langle\left\langle u_{\theta}^{2}\right\rangle\right\rangle-\left(2 u_{0}\left\langle u_{\theta}^{2}\right\rangle+2 u_{\pi}\left\langle u_{\theta} u_{\pi-\theta}\right\rangle-u_{0}\left\langle u_{\theta} u_{\pi-\theta}\right\rangle-u_{\pi}\left\langle u_{\theta}^{2}\right\rangle\right) \ln \frac{E_{F}}{T}\right] \frac{6 \zeta(3) T}{\pi v_{F}^{2}}(7)
\end{aligned}
$$

Next, we compute independently the static interaction vertices $\Gamma(\mathbf{k}, \mathbf{k})$ and $\Gamma(\mathbf{k},-\mathbf{k})\left(\Gamma^{k}\right.$ in the Fermi liquid notations) to second order in $U(q)$, including the renormalizations in the particle-hole and particle-particle channels. Collecting both contributions, we find

$$
\begin{aligned}
\frac{m}{2 \pi} \Gamma(\mathbf{k}, \mathbf{k}) & =\tilde{u}_{0}+\left\langle\left\langle u_{\theta}^{2}\right\rangle\right\rangle-\left\langle u_{\theta}^{2}\right\rangle \ln \frac{E_{F}}{T} \\
\frac{m}{2 \pi} \Gamma(\mathbf{k},-\mathbf{k}) & =\tilde{u}_{\pi}+\left\langle u_{\theta} u_{\pi-\theta}\right\rangle\left(1-\ln \frac{E_{F}}{T}\right) .
\end{aligned}
$$

where

$$
\begin{aligned}
& \tilde{u}_{0}=u_{0}\left(1-2 u_{0}+2\left\langle u_{\theta}\right\rangle\right), \\
& \tilde{u}_{\pi}=u_{\pi}\left(1-2 u_{\pi}\langle\langle 1\rangle\rangle+2\left\langle\left\langle u_{\theta}\right\rangle\right\rangle\right) .
\end{aligned}
$$

The charge and spin components $f_{c, s}(\pi)$ are obtained using Eq. (2). Evaluating the expression $f_{c}^{2}(\pi)+3 f_{s}^{2}(\pi)$ to third order in $U(q)$ and substituting the result into Eq.(11), we find that Eq. ( (7) is fully reproduced. Therefore, at least to third order, the prefactor of the $T$ term in $\delta \gamma(T)$ is expressed via exact scattering amplitudes, which include the renormalizations in the Cooper channel.

At low $T$, the particle-particle renormalizations are more relevant that those in the particle-hole channel, as the former contain a large factor of $\ln E_{F} / T$. Keeping only the particle-particle renormalization, we obtain

$$
\begin{aligned}
& f_{c}(\pi)=2 u_{0}-u_{\pi}+\left(\left\langle u_{\theta} u_{\pi-\theta}\right\rangle-2\left\langle u_{\theta}^{2}\right\rangle\right) \ln \frac{E_{F}}{T} \\
& f_{s}(\pi)=-\left(u_{\pi}-\left\langle u_{\theta} u_{\pi-\theta}\right\rangle \ln \frac{E_{F}}{T}\right)
\end{aligned}
$$

We see that both $f_{s}(\pi)$ and $f_{c}(\pi)$ contain logarithmic corrections. For $u_{\theta}=$ const $\equiv u$, Eqs. (10) reduce to

$$
f_{c}(\pi)=-f_{s}(\pi)=u\left(1-u \ln \frac{E_{F}}{T}\right) \approx \frac{u}{1+u \ln \frac{E_{F}}{T}}
$$

A better estimate is obtained if we assume, following AE, a simple model form for the irreducible interaction in the particle-particle channel: $J$

$$
J^{C}(q)=\Gamma(k,-k ; k+q,-k-q)=a w /\left(q^{2}+a^{2}\right),
$$

where $q=2 k_{F} \sin \theta / 2$ and $w$ has the units of velocity. The partial components of $J^{c}(q)$ are

$$
J_{n}^{C}=\frac{w e^{-\beta n}}{\sqrt{a^{2}+4 k_{F}^{2}}}, \quad \beta=2 \ln \left(\frac{a}{2 k_{F}}+\sqrt{1+\frac{a^{2}}{4 k_{F}^{2}}}\right)
$$


In the limit of $T \rightarrow 0$, the sums over $n$ in Eq. (3) for $\Gamma^{C}(0)$ and $\Gamma^{C}\left(2 k_{F}\right)$ are dominated by large $n$; replacing summation over $n$ by integration, we obtain

$$
\Gamma^{C}(0)=\frac{2 \pi}{m} \frac{\ln L}{\beta L}, \Gamma^{C}\left(2 k_{F}\right)=\frac{2 \pi}{m} \frac{1-e^{-\beta}}{\beta L},
$$

where $L=\ln E_{F} / T$. In this limit, $\Gamma^{C}(0)$ is larger than $\Gamma^{C}\left(2 k_{F}\right)$ by $\ln L$, hence $f_{c}(\pi) \approx 2 \ln L /(\beta L) \gg f_{s}(\pi)$, and the full result for the specific heat coefficient becomes

$$
\delta \gamma(T)=-\frac{3 \zeta(3)}{2 \pi\left(v_{F}^{*}\right)^{2}}\left[\frac{2 \ln \ln \frac{E_{F}}{T}}{\beta \ln \frac{E_{F}}{T}}\right]^{2} T .
$$

Note that the prefactor depends on the functional form of $\Gamma^{C}(q)$ but not on the magnitude of the interaction. Eq. (13) is valid only at such low temperatures that $\ln L \gg$ 1. For a more realistic case of $L \gtrsim 1$, both $\Gamma^{C}(0)$ and $\Gamma^{C}\left(2 k_{F}\right)$ are of order $1 / L$, and $\delta \gamma(T) \propto T /\left(\ln E_{F} / T\right)^{2}$.

A more fundamental reason why the ultra-low $T$ regime is unaccessible is the Kohn-Luttinger effect: the superconducting instability for a nominally repulsive interaction 22]. It is known that irreducible $J^{C}(q)$ is nonanalytic near $q=2 k_{F}$ due to screening of the original pairing interaction by the particle-hole excitations. Screening produces a long-range component of $J^{C}(q)$. In 2D, the Kohn-Luttinger effect is a bit tricky as one needs to include vertex corrections to the polarization bubble to obtain an oscillating long-range component of the pairing interaction $\sin \left(2 k_{F} r\right) / r^{2}$ between particles at the Fermi surface [23]. Because of oscillations and $1 / r^{2}$ behavior, the partial harmonics $J_{n}^{C}$ acquire negative parts that fall off with $n$ algebraically rather then exponentially: $J_{n}^{C, K L} \approx-\alpha / n^{2}, \alpha>0$ (at small $U, \alpha \propto U^{3}$ ). As a result, $J_{n}^{C}$ become negative for $n>n_{c}$, which implies superconductivity. For a moderately strong interaction $[m U(q) \gtrsim 1], J_{n}^{C}$ becomes negative already for $n=1$ ( $\left|J_{1}^{C}\right|$ is the largest), and the system becomes unstable towards $p$-wave pairing at $T_{p}$ defined by $(m \alpha / 2 \pi) \ln E_{F} / T_{p}=1$. Near $T_{p}$, the $n=1$ term dominates the sums in Eq. (3), both $\Gamma^{C}(0)$ and $\Gamma^{C}\left(2 k_{F}\right)$ diverge as $\alpha /(1-(m \alpha / 2 \pi) L) \propto 1 /\left(T-T_{p}\right)$, hence

$$
\delta \gamma(T)=-\frac{36 \zeta(3)}{2 \pi\left(v_{F}^{*}\right)^{2}}\left(\frac{T_{p}}{T-T_{p}}\right)^{2} T .
$$

To verify our computational procedure, we also consider the $1 \mathrm{D}$ case. The procedure that we used in $2 \mathrm{D}$ is also applicable to the $1 \mathrm{D}$ case, with the only modification that angular averages of the interaction in the particle-hole channel [Eq. (6)] are replaced by a sum of just two terms, for $\theta=0$ and $\theta=\pi$, as the Fermi surface in $1 \mathrm{D}$ consists of just two points $k= \pm k_{F}$. The integrand in (6) vanishes at $\theta=\pi$ and diverges logarithmically at $\theta=0$, i.e., for $D=1$, the renormalization of the interaction in the particle-hole channel also leads to logarithmic corrections:

$$
\left\langle\left\langle u_{\theta}\right\rangle\right\rangle \rightarrow u_{0} \ln \frac{E_{F}}{T}, \quad\left\langle\left\langle u_{\theta}^{2}\right\rangle\right\rangle \rightarrow u_{0}^{2} \ln \frac{E_{F}}{T}, \quad\langle\langle 1\rangle\rangle \rightarrow \ln \frac{E_{F}}{T},
$$

where now $u_{\theta}=U\left(2 k_{F} \sin \theta / 2\right) /\left(2 \pi v_{F}\right)$. Combining the logarithms in the particle-particle and particle-hole channel and neglecting non-logarithmic second-order terms, we reproduce the well-known results for the charge- and spin scattering amplitudes in 1D [16, 17, 18]:

$$
f_{c}(\pi)=2 u_{0}-u_{\pi}, f_{s}(\pi) \approx-\frac{u_{\pi}}{1+2 u_{\pi} \ln \frac{E_{F}}{T}}
$$

The logarithmic corrections are cancelled out in $f_{c}(\pi)$, but are present in $f_{s}(\pi)$. The interplay between the behavior of the renormalized spin amplitude and the specific heat in $1 \mathrm{D}$ is a more subtle issue, because the backscattering part of $\delta \gamma(T)$ in $1 \mathrm{D}$ contains extra $O(T)$ terms and does not reduce to Eq. (1) with the renormalized $f_{s}(\pi)$ [15, 24, 25].

To elucidate the difference between 2D and 1D further, we consider a $2 \mathrm{D}$ system with a non-circular Fermi surface. Near an arbitrary point $\mathbf{k}_{F}$ on such a surface, the fermionic dispersion can be expanded as

$$
\epsilon_{\mathbf{k}}=v_{F} k_{\|}+\frac{k_{\perp}^{2}}{2 m_{c}}
$$

where $k_{\|}$and $k_{\perp}$ are the projections of vector $\mathbf{k}-\mathbf{k}_{F}$ on the normal and tangent to the Fermis surface at point $\mathbf{k}_{F}$, correspondingly, $v_{F}$ is the local value of the Fermi velocity, and $m_{c}=1 / \kappa v_{F}$ is related to the local curvature, $\kappa$, of the Fermi surface [13, 26]. For a circular Fermi surface, $\kappa=k_{F}^{-1}$ so that $m_{c}=m=k_{F} / v_{F}$ and our $2 \mathrm{D}$ results are valid. If $m_{c} \gg m$, the dispersion near two symmetric points $\pm \mathbf{k}_{F}$ is almost one-dimensional and, if only $u_{0}$ and $u_{\pi}$ are relevant, we should reproduce $1 \mathrm{D}$ results. Indeed, evaluating the particle-hole contributions, labelled above as $\langle\langle\ldots\rangle\rangle$, for the case of $m \gg m_{c}$, we find that they have the same logarithmic behavior, as in Eq.(16); the only difference being that the logarithm is now cut by the largest of the two energies: $T$ and $E_{c}=k_{F}^{2} / 2 m_{c}$. Substituting these results into the expression for $f_{c}(\pi)$, we find that to logarithmic accuracy

$$
f_{c}(\pi)=2 u_{0}-u_{\pi}-2\left(u_{0}^{2}+u_{\pi}^{2}-u_{0} u_{\pi}\right) \ln \max \left(E_{c} / T, 1\right)
$$

For $m_{c}=\infty, E_{c}=0$, and the logarithmic term vanishes, just as it happens in 1D.

We see that the curvature of the Fermi surface is the crucial element of $2 \mathrm{D}$ consideration [27]. When the curvature is finite, the particle-hole renormalizations of the scattering ampludes and of $\delta \gamma(T)$ are not logatithmic at the smallest $T$, and the logarithms only come from particle-particle renormalizations. Then $f_{s}(\pi)$, $f_{s}(\pi)$, and $\delta \gamma(T)$ are all logarithmically reduced. When 
the curvature is zero, the dispersion is one-dimensional, particle-hole renormalizations also become logarithmic, and for $f_{c}(\pi)$ the logarithms from the particle-particle and particle-hole renormalizations are cancelled out. In the eikonal approximation used by $\mathrm{AE}$ and in earlier 2D bosonization theories [28], the curvature of the Fermi surface is neglected. In this situation, the charge amplitude behaves as in $1 \mathrm{D}$ and is not renormalized, and $\delta \gamma(T)$ remains linear in $T$.

To summarize, in this paper we analyzed the effect of the Cooper-channel renormalization on the temperature dependence of the specific heat. We have shown the nonanalytic term in the specific heat coefficient of a 2D Fermi liquid is expressed via the square of the full backscattering amplitude $f(\pi)$, renormalized in both particlehole and particle-particle channels. Due to the particleparticle renormalization, both the charge and spin components of $f(\pi)$ are reduced by a factor of $\ln \left(E_{F} / T\right)$ in the limit of $T \rightarrow 0$. Consequently, the temperature dependence of $\delta \gamma(T)$ is $T S(T) / \ln ^{2}\left(E_{F} / T\right)$, where $S(T)$ is a slowly varying function, whose form depends on the details of the interaction in the particle-particle channel. When applied to 1D, our method reproduces the cancellation between particle-hole and particle-particle contributions to the charge channel. The logarithmic renormalization of the charge amplitude is due to a specifically higher-dimensional effect-a finite curvature of the Fermi surface. For a flat Fermi surface, this renormalization is absent.

We acknowledge helpful discussions with I. L. Aleiner, A. M. Finkelstein, L. I. Glazman, K. B. Efetov, A. A. Nersesyan, R. Saha and G. Schwiete, support from nsf-dmr 0604406 (A. V. Ch.), 0308377 (D. L. M.), and the hospitality of the Aspen Center of Physics.

[1] C.J. Pethick and G.M. Carneiro, Phys. Rev. A 7, 304 (1973) and references therein.

[2] D. Belitz, T. R. Kirkpatrick, and T. Vojta, Rev. Mod. Phys. 77, 579 (2005) and references therein.

[3] D. Coffey and K. S. Bedell, Phys. Rev. Lett. 71, 1043 (1993).

[4] M. A. Baranov, M. Yu. Kagan, and M. S. Mar'enko, JETP Lett. 58, 709 (1993).

[5] G. Y. Chitov and A. J. Millis, Phys. Rev. Lett. 86, 5337 (2001).
[6] A. V. Chubukov and D. L. Maslov, Phys. Rev. B 68, 155113 (2003); Phys. Rev. B 74, 079907 (2006).

[7] J. Betouras, D. Efremov, and A. Chubukov, Phys. Rev. B 72, 115112 (2005).

[8] G. Schwiete and K. B. Efetov, Phys. Rev. B 74, 165108 (2006).

[9] A. Shekhter and A.M. Finkelstein, Phys. Rev. B 74, 205122 (2006); Proc. Nat. Acad. Sci. 103 (2006) 15765; Proc. Nat. Acad. Sci. 103 (2006) 18874.

[10] D. L. Maslov, A. V. Chubukov, and R. Saha, Phys. Rev. B 74, 220402 (2006).

[11] A. V. Chubukov, D. L. Maslov, S. Gangadharaiah, and L. I. Glazman, Phys. Rev. Lett. 95, 026402 (2005); Phys. Rev. B 71, 205112 (2005).

[12] A. V. Chubukov, D.L. Maslov, and A. J. Millis, Phys. Rev. B 73, 045128 (2006).

[13] A.V. Chubukov and A.J. Millis, Phys. Rev. B 74, 115119 (2006).

[14] The spin susceptibility both in $2 \mathrm{D}$ and $3 \mathrm{D}$, as well as the specific heat in $3 \mathrm{D}$, contain other contributions which do not reduce to $1 \mathrm{D}$ scattering [9, 10]].

[15] I. L. Aleiner and K. B. Efetov, Phys. Rev. B 74, 075102 (2006); cond-mat/0610345 Notice that the "backscattering amplitude" is defined in these papers as the irreducible amplitude, without the renormalizations in the Cooper channel.

[16] Yu. A. Bychkov, L. P. Gor'kov, and I. E. Dzyaloshisnkii, Sov. Phys. JETP 23, 489 (1966).

[17] J. Solóyom, Adv. Phys. 28, 209 (1979).

[18] V. J. Emery, in Highly Conducting One-Dimensional Solids, eds. J. T. Devreese, R. E. Evrard, and V. E. van Doren, (Plenum Press, New York, 1979), p. 247.

[19] H. J. Schulz, in Mesoscopic Quantum Physics, Les Houches XXI (eds. E. Akkermans, G. Montambaux, J. L. Pichard, and J. Zinn-Justin), (Elsevier, Amsterdam, 1995), p. 533; G. I. Japaridze and A. A. Nersesyan, Phys. Lett. 94 A, 224 (1983).

[20] E.M. Lifshitz and L.P. Pitaevskii, Statistical Physics, p.2, Pergamon Press, 1996.

[21] We corrected a sign error in $\Xi_{3 a}$ in Ref. [11].

[22] W. Kohn and J. Luttinger, Phys. Rev. Lett. 15, (1965).

[23] A. Chubukov, Phys. Rev. B 48, 1097-1104 (1993)

[24] S. Lukyanov, Nucl. Phys. B 522, 533 (1998).

[25] We thank I. Aleiner and K. Efetov for pointing out a mistake in our original treatment of the 1D case.

[26] Out of all second-order terms we keep in Eq.(17) the one that can be comparable to the first-order term $\left(v_{F} k_{\| \mid}\right)$.

[27] For a related discussion on the role of the Fermi surface curvature in 2D, see A. Chubukov and D. Khveshchenko, Phys. Rev. Lett. 97, 226403 (2006).

[28] see. e.g., M. J. Lawler et al., Phys. Rev. B73, 085101 (2006) and references therein. 